\title{
Directrices para la remediación de suelos contaminados con hidrocarburos
}

\author{
José Guadalupe Chan-Quijano* \\ Aarón Jarquín-Sánchez \\ Susana Ochoa-Gaona \\ Pablo Martínez-Zurimendi \\ El Colegio de la Frontera Sur \\ Leonardo Noriel López-Jiménez \\ Parque Nacional Arrecifes de Xcalak, \\ Comisión Nacional de Áreas Naturales Protegidas \\ Alejandra Lázaro-Vázquez \\ El Colegio de la Frontera Sur
}

\section{Resumen}

Las directrices para la remediación de suelos son recomendaciones generales orientadas al empleo de biotecnologías que han demostrado su capacidad para el saneamiento y la recuperación de suelos contaminados con hidrocarburos totales de petróleo. Con base en una revisión bibliográfica, se explican los mecanismos biológicos, ecológicos, económicos y sociales que sustentan la capacidad remediadora de diversas técnicas, lo que permite seleccionar los métodos adecuados de acuerdo con el nivel de afectación. En este trabajo, se presentan los datos requeridos para fijar las directrices y elegir los mecanismos de remediación de los suelos contaminados con hidrocarburos totales de petróleo.

\section{Palabras clave}

Gestión de suelos contaminados, fitorremediación, biotecnologías, recomendaciones, hidrocarburos.

Recibido: 14/07/2014 · Aceptado: 11/08/2014

Correo electrónico: jchan@ecosur.edu.mx·ajarquin@ecosur.mx 


\title{
Guidelines for the remediation of contaminated soil with oil
}

\author{
José Guadalupe Chan-Quijano* \\ Aarón Jarquín-Sánchez \\ Susana Ochoa-Gaona \\ Pablo Martínez-Zurimendi \\ El Colegio de la Frontera Sur \\ Leonardo Noriel López-Jiménez \\ Parque Nacional Arrecifes de Xcalak, \\ Comisión Nacional de Áreas Naturales Protegidas \\ Alejandra Lázaro-Vázquez \\ El Colegio de la Frontera Sur
}

\begin{abstract}
The guidelines for soil remediation include general recommendations for the use of biotechnologies that have demonstrated their ability for sanitation and recovery of soil contaminated with total petroleum hydrocarbons. Based on a bibliographic review the biological, ecological, economic and social mechanisms that sustain a remedial level are explained to allow the selection of the best technology according to the level of contamination. The data required to establish guidelines and mechanisms for selecting remediation of soils contaminated with total petroleum hydrocarbons are presented in this paper.
\end{abstract}

\section{KEY WORDS}

Management of contaminated soils, phytoremediation, biotechnologies, recommendations, hydrocarbons. 


\section{Introducción}

El desarrollo económico y social de un país lleva implícito el uso de energía, el cual, hasta ahora, se ha basado en el empleo de derivados del petróleo. Sin embargo, las biotecnologías que permiten la extracción, conducción y transformación del petróleo han producido la contaminación del suelo por accidentes de derrames de tanques de almacenamiento, recortes de perforación, pozos, baterías de separación, complejos procesadores de gas, centrales de almacenamiento y bombeo, ruptura o daños de oleoductos, red de ductos y presas para el confinamiento de desechos sólidos y líquidos procedentes de la perforación y mantenimiento de los pozos petroleros (Chiou, McGroddy y Kile, 1998; RiveraCruz, 2004; Chan-Quijano et al., 2012).

Cuando los seres humanos tengamos plena conciencia de que estamos afectando los servicios ambientales y el equilibrio dinámico de los ecosistemas comenzaremos a buscar alternativas más sofisticadas para remediar las áreas vulneradas, sobre todo a causa de la contaminación por derrames de hidrocarburos en los suelos, pues estos derrames son un factor de reducción de la sostenibilidad del ambiente (Abraham, 2004). La búsqueda de soluciones debe profundizarse en el corto plazo para restaurar los suelos dañados.

Las experiencias de remediación de suelos en México muestran que la decisión de aplicar la biotecnología apropiada requiere del conocimiento previo del suelo a remediar, del tipo de contaminantes y de sus niveles de concentración (IPIECA, 1991: 15; CCME, 2001: 8). No obstante, aún no se cuenta con la información que explique la forma en que se comportan estas biotecnologías en las distintas zonas del país, en las que las permeabilidades son bajas y los grupos del suelo y las condiciones climáticas difieren.

El suelo es un recurso natural de renovación muy lenta, contiene nutrimentos, minerales, compuestos orgánicos y organismos vivos, y posee la capacidad de soportar el crecimiento de las plantas (Plaster, 2000). La contaminación del suelo por hidrocarburos es una problemática muy vasta, pues no solo repercute en el uso sustentable del suelo y el desarrollo urbano, sino que también tiene consecuencias en la salud de la población y, en la mayoría de los casos, en otros recursos naturales como el agua. Por ello, en México, al igual que en muchos países, es importante prevenir la contaminación del suelo, y su recuperación es una prioridad (González-Chávez, 2005). 
En México, la Ley General del Equilibrio Ecológico y la Protección al Ambiente (LGEEPA), establece que quien contamine el suelo debe restaurarlo, pero no plantea disposiciones con carácter normativo, ni define quién habrá de regular esta materia, ni cómo lo haría (Carmona-Díaz, 2012; Presidencia de la República, 1996: 27). Pese a lo anterior, Carabias-Lillo (1999) menciona que el problema de la contaminación de los suelos por derrames de petróleo no siempre está relacionado con su marco conceptual, con sus herramientas metodológicas o con el tipo de hipótesis que propone, ni tampoco con las acciones específicas que de él se derivan. Es por ello que las técnicas para la remediación de suelos contaminados ofrecen orientación práctica para restaurar los sitios afectados y sus particularidades.

El presente trabajo pretende analizar, con ayuda de la literatura publicada, las directrices, recomendaciones y mecanismos de remediación de los suelos contaminados con hidrocarburos del petróleo para, de acuerdo con el grado de afectación, poder seleccionar la tecnología más conveniente para su posible aplicación.

\section{Los tres ejes de la sustentabilidad para la remediación de suelos contaminados por hidrocarburos}

Las directrices se presentan una vez seleccionadas las recomendaciones apropiadas para intervenir de acuerdo con el grado de contaminación correspondiente. En lo fundamental, estas directrices ayudan a asegurar que la remediación de los suelos contaminados sea ambientalmente eficaz, metodológica y económicamente eficiente y socialmente aceptada (Dirección General de Parques Nacionales y Agencia de Parques de Canadá, 2008: 97).

Los tres ejes de la sustentabilidad (cuadro 1) identifican de forma integrada y jerarquizada tanto los problemas que enfrenta una institución o empresa como su entorno en los campos ambiental, social y económico, considerando que las acciones que deben realizar sean compatibles con la filosofía del desarrollo sustentable (IMP, 2010: 38). 
Cuadro 1. Los tres ejes de la sustentabilidad

\begin{tabular}{ll}
\hline Sustentabilidad ecológica & $\begin{array}{l}\text { Los ecosistemas deben mantener, a través del } \\
\text { tiempo, las características fundamentales para } \\
\text { su supervivencia en cuanto a componentes e } \\
\text { interacciones. }\end{array}$ \\
& Implica que el sistema bajo uso produce una \\
Sustentabilidad económica & dentabilidad razonable y estable en el transcurso \\
& dicho manejo. \\
Sustentabilidad social & Aspira a que la forma de manejo y de organi- \\
& zación social permita un grado aceptable de \\
& satisfacción de las necesidades de la población \\
& que usa el recurso. El manejo sustentable puede, \\
por lo tanto, significar distintas cosas en cada & momento histórico de un país. \\
\hline
\end{tabular}

Fuente: Etchevers (1999).

En los últimos años se ha observado un significativo deterioro en el ambiente, reflejado en extensas áreas de suelos contaminados por hidrocarburos, de manera señalada en los estados del sureste del país: Veracruz, Chiapas, Tabasco y Campeche (Ochoa-Gaona et al., 2011: 144). En este sentido, IbáñezPérez (2012) menciona que es necesario crear modelos de sustentabilidad que incluyan nuevos criterios espaciales, culturales, sociales y políticos de las áreas afectadas. Al integrar indicadores de sustentabilidad más acordes con el tipo de contaminación se obtiene información fehaciente sobre el estado de las tres dimensiones del desarrollo sustentable, lo que permite fundamentar con mayor precisión las políticas encaminadas a mejorar la remediación de los suelos contaminados.

Por ello, a partir de una perspectiva de sustentabilidad, Saval (1999) establece criterios para elegir una técnica de remediación, los cuales competen no sólo a las técnicas de remediación, sino también a las características del sitio. A continuación enumeramos algunos de estos criterios:

- Comprender la función que desempeña el suelo afectado en el sitio específico. 
- Conocer perfectamente el problema de contaminación con información reciente.

- Identificar las características de una técnica de remediación.

- Conocer las ventajas, desventajas y limitaciones de las técnicas de remediación disponibles.

- Definir con claridad el uso que se le dará al suelo (agrícola, forestal, recreativo, residencial, de conservación, comercial o industrial) después de su remediación, en estricto apego a la legislación ambiental mexicana.

\section{Orientaciones y criterios para seleccionar la técnica adecuada y como aplicarla}

La contaminación de los suelos suele ser un problema global y, por ende, se han encontrado biotecnologías para la remediación de los sitios impactados (Moosavi y Seghatoleslami, 2013). Así, se dispone de técnicas de retención, extracción o separación y destrucción (cuadro 2).

Cuadro 2. Clasificación de técnicas de remediación con base en su efecto sobre los contaminantes

Retención

Extracción o separación

Destrucción
Confinamiento en celdas, barreras impermeables, fijación.

Filtración por carbón activado, lavado con agentes tensoactivos, extracción de producto libre, extracción de vapores.

Biorremediación, fitorremediación, incineración.

Fuente: Saval (1999).

Para Saval (1999) la clasificación más común de la perspectiva del riesgo y del saneamiento de la zona contaminada debe fundarse en las propiedades biológicas, físico-químicas y térmicas de los suelos, lo que corresponde al principio del funcionamiento de la clasificación (cuadro 3). 
Cuadro 3. Clasificación de técnicas de remediación

de acuerdo con el principio de su funcionamiento

\begin{tabular}{ll}
\hline Biológicas & Biorremediación, fitorremediación. \\
Físico-químicas & Solidificación, estabilización, extracción de vapores. \\
Térmicas & Desorción por inyección de vapor, incineración. \\
\hline Fuente: Saval (1999) &
\end{tabular}

Fuente: Saval (1999).

En la práctica, las dos clasificaciones (cuadros 2 y 3) son válidas e incluso complementarias, ya que por lo general es menester combinarlas (Saval, 1999). Pero, además, los especialistas del área de remediación de suelos utilizan el concepto de la atenuación natural. Saval (1999) indica que esta atenuación se refiere al trabajo que la naturaleza hace por sí misma para degradar los contaminantes a través de los ciclos biogeoquímicos que llevan a cabo los microorganismos nativos del suelo, sin la intervención del ser humano. Este concepto se conoce también como biorremediación intrínseca. Empero, el tiempo que necesita la naturaleza para recuperarse puede variar de unos pocos días a más de diez años.

\section{Técnicas biológicas empleadas para la remediación de suelos contaminados por hidrocarburos}

Existen diversas técnicas, mas los tratamientos biológicos o biorremediación tienen como sustento favorecer los procesos microbiológicos que de forma natural se producen en el suelo y que conllevan la degradación del contaminante (Sims, 1993; Fernández-Linares et al., 2006: 179). El objetivo es conseguir la mineralización de los contaminantes, esto es, transformar los compuestos químicos nocivos en compuestos inocuos o menos tóxicos, tales como dióxido de carbono, agua o materia celular (cuadro 4). 


\section{Cuadro 4. Biotecnologías utilizadas en la remediación de suelos contaminados}

\begin{tabular}{|c|c|}
\hline Bioestimulación & $\begin{array}{l}\text { Implica la adición de oxígeno y/o nutrientes al suelo contaminado con el fin } \\
\text { de estimular la actividad de los microorganismos autóctonos y así propiciar la } \\
\text { biodegradación de los contaminantes. }\end{array}$ \\
\hline Bioaumentación & $\begin{array}{l}\text { Consiste en añadir microorganismos vivos que tienen la capacidad de degradar } \\
\text { el contaminante en cuestión y así promover su biodegradación o biotransfor- } \\
\text { mación. }\end{array}$ \\
\hline Biolabranza & $\begin{array}{l}\text { Radica en airear por cúmulos el suelo contaminado, y agregar agua y fertilizan- } \\
\text { tes; los microorganismos que se encuentran en este suelo ya están adaptados a él } \\
\text { y -al recibir oxígeno a través del aire, agua y fertilizantes en cantidades precisas } \\
\text { y con el ambiente y temperatura adecuados- degradarán el hidrocarburo. }\end{array}$ \\
\hline Bioventeo & $\begin{array}{l}\text { Estriba en estimular la biodegradación aerobia de un contaminante por medio } \\
\text { del suministro de aire en el sitio contaminado, a través de pozos de inyección o } \\
\text { extracción en la zona no saturada por el derrame, y, si es necesario, se le añaden } \\
\text { nutrientes. }\end{array}$ \\
\hline Biorreactores & $\begin{array}{l}\text { Es la tecnología más adecuada, pues optimiza el uso de los microorganismos. } \\
\text { Permite la combinación controlada y eficiente de procesos químicos, físicos y } \\
\text { biológicos que mejoran y aceleran la biodegradación. Uno de los reactores más } \\
\text { utilizados para biorremediar suelos es el biorreactor de lodos, en él el suelo con- } \\
\text { taminado se mezcla constantemente con un líquido, y la degradación se lleva a } \\
\text { cabo en la fase acuosa por microorganismos en suspensión o inmovilizados en } \\
\text { la fase sólida. }\end{array}$ \\
\hline $\begin{array}{l}\text { Biodegradación } \\
\text { en fase sólida }\end{array}$ & $\begin{array}{l}\text { Este tipo de tecnología, por lo general, se aplica ex situ e incluye el composteo, } \\
\text { las pilas estáticas y las pilas alargadas. Es decir, el material contaminado se mez- } \\
\text { cla con abonos orgánicos (paja, aserrín, estiércol, desechos agrícolas), pues sus } \\
\text { características contribuyen a recobrar el balance de nutrientes y aseguran una } \\
\text { mejor aireación y la generación del calor durante el proceso. Las pilas estáticas } \\
\text { (biopilas) son una forma de composteo que añade al sistema agua y nutrientes, } \\
\text { y se colocan en áreas de tratamiento (que incluyen alguna forma de aireación y } \\
\text { sistemas para colectar lixiviados). Lo usual es que las pilas de suelo se cubran } \\
\text { con plástico para controlar los lixiviados, la evaporación y la volatilización de } \\
\text { contaminantes, además de que así se favorece su calentamiento. }\end{array}$ \\
\hline Fitorremediación & $\begin{array}{l}\text { Es una tecnología in situ, no destructiva, de bajo costo, para limpiar suelos con- } \\
\text { taminados con petróleo; se basa en el uso de plantas que tienen la propiedad de } \\
\text { acumular metales pesados y disminuir la concentración de hidrocarburos. Esta } \\
\text { técnica estimula la actividad microbiana en la rizosfera para degradar los conta- } \\
\text { minantes de los suelos, consiguiendo la remoción, transferencia, estabilización y } \\
\text { neutralización de compuestos orgánicos e inorgánicos tóxicos. }\end{array}$ \\
\hline
\end{tabular}

Fuente: Velasco-Trejo y Volke-Sepúlveda (2003); Merkl, Schultze-Kraft e Infante (2004); Sierra- 
La fitorremediación forma parte de la biorremediación; puede aplicarse a contaminantes orgánicos presentes en sustratos sólidos, líquidos o en el aire, y lo hace utilizando sus propias fitotecnologías (cuadro 5).

Cuadro 5. Fitotecnologías utilizadas en la fitorremediación de suelos contaminados

\begin{tabular}{|ll}
\hline Fitoextracción & $\begin{array}{l}\text { Uso de plantas acumuladoras de elementos tóxicos o com- } \\
\text { puestos orgánicos para retirarlos del suelo mediante su absor- } \\
\text { ción y concentración en las partes cosechables. }\end{array}$ \\
\hline Fitoestabilización & $\begin{array}{l}\text { Uso de plantas para reducir la biodisponibilidad de los conta- } \\
\text { minantes en el entorno, mejorando las propiedades físicas y } \\
\text { químicas del medio. }\end{array}$ \\
\hline Fitoinmovilización & $\begin{array}{l}\text { Uso de las raíces de las plantas para fijar o inmovilizar los } \\
\text { contaminantes en el suelo. Junto con la anterior, son técnicas } \\
\text { de contención. }\end{array}$ \\
\hline Fitovolatilización & $\begin{array}{l}\text { Uso de plantas para eliminar los contaminantes del medio a } \\
\text { través de su volatilización, y para eliminar contaminantes del } \\
\text { aire. }\end{array}$ \\
\hline Fitodegradación & $\begin{array}{l}\text { Uso de plantas y microorganismos asociados para degradar } \\
\text { contaminantes orgánicos. }\end{array}$ \\
\hline Rizofiltración & $\begin{array}{l}\text { Uso de raíces para absorber contaminantes del agua y de otros } \\
\text { efluentes acuosos. }\end{array}$ \\
\hline
\end{tabular}

Fuente: Cunningham, Berti y Huang (1995); Fernández-Linares et al. (2006: 179); Carpena y Bernal (2007); Moosavi y Seghatoleslami (2013).

Por lo tanto, la fitorremediación consiste en utilizar principalmente plantas en la interacción del efecto rizosfera con el suelo y los microorganismos (Frély, 2013: 229).

En una caracterización de 12 sitios contaminados por hidrocarburos realizada por Ochoa-Gaona et al. (2011: 144) se reportan 45 especies nativas, pertenecientes a 23 familias botánicas, que crecen y se desarrollan en áreas contaminadas con petróleo en el estado de Tabasco (cuadro 6). 


\section{Cuadro 6. Listado de especies arbóreas en los 12 sitios inventariados ( $1000 \mathrm{~m}^{2}$ por sitio)}

\begin{tabular}{|c|c|c|c|c|c|}
\hline Núm. & Especie & Familia & Núm. & Especie & Familia \\
\hline 1 & Acacia cornigera & Fabaceae & 24 & Lonchocarpus hondurensis & Fabaceae \\
\hline 2 & Acoelorrhaphe wrightii & Arecaceae & 25 & Mangifera indica & Anacardiaceae \\
\hline 3 & Andira galeottiana & Fabaceae & 26 & Miconia albicans & Fabaceae \\
\hline 4 & Andira inermis & Fabaceae & 27 & Miconia argentea & Melastomataceae \\
\hline 5 & Annona reticulata & Annonaceae & 28 & Miconia mexicana & Melastomataceae \\
\hline 6 & Bursera simaruba & Burseraceae & 29 & Mimosa albida & Fabaceae \\
\hline 7 & Byrsonima crassifolia & Malpighiaceae & 30 & Mimosa pigra & Fabaceae \\
\hline 8 & Casearia sylvestris & Salicaceae & 31 & Myrica cerifera & Myricaceae \\
\hline 9 & Cecropia obtusifolia & Urticaceae & 32 & Pachira aquatica & Malvaceae \\
\hline 10 & Citharexylum hexangulare & Verbenaceae & 33 & Parmentiera aculeata & Bignoniaceae \\
\hline 11 & Citrus aurantium & Rutaceae & 34 & Pithecellobium lanceolatum & Fabaceae \\
\hline 12 & Citrus limon & Rutaceae & 35 & Posoqueria latifolia & Rubiaceae \\
\hline 13 & Citrus sinensis & Rutaceae & 36 & Psidium guajava & Myrtaceae \\
\hline 14 & Coccoloba barbadensis & Polygonaceae & 37 & Randia aculeata & Rubiaceae \\
\hline 15 & Cocos nucifera & Arecaceae & 38 & Sabal mexicana & Arecaceae \\
\hline 16 & Combretum fruticosum & Combretaceae & 39 & Scheelea liebmannii & Arecaceae \\
\hline 17 & Crataeva tapia & Capparaceae & 40 & Solanum erianthum & Solanaceae \\
\hline 18 & Cupania dentata & Sapindaceae & 41 & Solanum hirtum & Solanaceae \\
\hline 19 & Davilla kunthii & Dilleniaceae & 42 & Spondias mombin & Anacardiaceae \\
\hline 20 & Eugenia capuli & Myrtaceae & 43 & Tabebuia rosea & Bignoniaceae \\
\hline 21 & Guazuma ulmifolia & Malvaceae & 44 & Tabernaemontana alba & Apocynaceae \\
\hline 22 & Inga jinicuil & Fabaceae & 45 & Thevetia ahouai & Apocynaceae \\
\hline 23 & Inga laurina & Fabaceae & & & \\
\hline
\end{tabular}

Fuente: Ochoa-Gaona et al. (2011: 144). 
Sin embargo, hay reportes de 129 especies de plantas, de 47 familias botánicas, que están siendo utilizadas para degradar hidrocarburos del petróleo (Chan-Quijano et al., 2013). ${ }^{1}$

La Agencia de Protección Ambiental de Estados Unidos (EPA, por sus siglas en inglés) propone siete criterios para evaluar las alternativas de remediación utilizadas para la mejora del ambiente afectado, estos son:

1. Protección a la salud humana y del ambiente.

2. Compatibilidad con las leyes ambientales y aceptación por las autoridades ambientales.

3. Efectividad y permanencia de la remediación a corto y largo plazo.

4. Reducción de toxicidad, movilidad o volumen del contaminante por medio del tratamiento.

5. Implementación.

6. Bajo costo.

7. Aceptación y visto bueno de la comunidad.

Lo anterior implica no solo entender los criterios que cada técnica tiene establecidos sino, además, analizar las diferentes opciones. Así, se podrá determinar el desempeño de cada alternativa e identificar cuál es la óptima, y, en consecuencia, hacer uso de ella (IPIECA, 1991: 15; EPA, 1997: 23).

El Instituto Mexicano del Petróleo (IMP) (2010: 38), en su diagnóstico para la remediación de suelos contaminados, explica que realizar una restauración ambiental requiere:

- Identificación. Consiste en seleccionar, identificar, inventariar y priorizar las áreas que presenten contaminación por hidrocarburos, con la finalidad de conocer a fondo el problema y afrontarlo elaborando un programa resultado de esa evaluación preliminar del área contaminada.

${ }^{1}$ Para mayor información relativa a la remediación de suelos por plantas, consúltese Adams-Schroeder y Castillo-Acost (2000); Rivera-Cruz y Trujillo-Narcía (2004); ChanQuijano et al. (2012), Rivera-Cruz, Maldonado-Chávez y Trujillo-Narcía (2012) y PérezHernández et al. (2013). 
- Investigación. Comprende al menos tres etapas: 1) planeación de las actividades de investigación y el diagnóstico del sitio; 2) evaluación de las alternativas de remediación; y 3) selección de la tecnología óptima para la restauración de ese sitio en particular. La fase de investigación es la parte medular, pues su propósito es conocer el desarrollo de cada uno de los trabajos aplicados para un proceso de remediación, de modo tal que cada etapa quede planeada a detalle y, así, obtener resultados satisfactorios.

Limpieza del sitio. Se refiere a las acciones ligadas al desarrollo de los trabajos de restauración, mismas que se llevarán a cabo con la tecnología seleccionada. Entre otras labores, pueden considerarse el diseño del proceso de remediación, las tareas de limpieza, operación y mantenimiento, así como la verificación de las acciones de limpieza. Durante esta fase deberá hacerse un estricto seguimiento de cada etapa del proceso propuesto por la compañía remediadora, con la finalidad de evitar desviaciones que repercutan de manera negativa en el proceso de remediación.

- Restauración del sitio con plantas. Antes de la restauración deben verificarse los objetivos planteados con la participación de las autoridades ambientales; de este modo, será posible trabajar con plantas nativas en el área afectada. Mediante el diseño de un plan de muestreo óptimo -elaborado con base en el contaminante y en los atributos geofísicos del sitio (Saval, 1999; imp, 2010: 38)-, durante la fase de investigación se determina el contaminante (tipo y concentración) presente en el sitio afectado. A partir de esta caracterización puede conocerse la magnitud del daño causado por la contaminación, el riesgo que se deriva de esta contaminación, y las necesidades de remediación.

D’Luna-Fuentes, Vargas-Mena y Amezcua y Negrete-Fernández (1999) establecen que la aplicación de los sistemas de información geográfica sirven de apoyo para registrar el deterioro del suelo afectado por hidrocarburos. Estas herramientas también son de gran ayuda para evaluar la remediación de los suelos contaminados por hidrocarburos. 


\section{Técnicas empleadas para evaluar riesgos por contaminación por hidrocarburos}

El riesgo es el daño-pérdida esperable a consecuencia de la acción de una amenaza sobre un bien, trátese de la vida humana, bienes económicos o el entorno natural (Hewitt, 1997: 36). Álvarez-Gordillo (2011: 121) explica que para plantear los procesos de gestión del riesgo no es suficiente analizar y conocer los efectos del desastre (derrames de petróleo); esto debe estar coordinado por las acciones de las empresas responsables y las leyes que regulan las zonas impactadas.

Las zonas afectadas deben examinarse desde el punto de vista del riesgo, y debe considerarse sus consecuencias ambientales y económicas, razón por la cual es indispensable realizar estudios con un enfoque social. Frausto-Martínez (2008) establece tres etapas del análisis de riesgo:

a) Análisis de factores de riesgo. Consiste en el estudio de la peligrosidad, de la exposición y de la vulnerabilidad a las que pudiesen estar sujetos los habitantes de las zonas afectadas por contaminación por hidrocarburos.

b) Evaluación del riesgo. Deben calcularse o estimarse las pérdidas ambientales esperables amén de compararse los criterios de fertilidad de los suelos dañados.

c) Análisis y medidas de mitigación del riesgo de la contaminación.

En el procedimiento de evaluación de riesgos, el IMP (2010: 38) establece que en los muestreos y las determinaciones analíticas es menester atender a:

1. La identificación del problema.

2. La evaluación de la exposición.

3. La evaluación de la toxicidad.

4. La caracterización o cuantificación del riesgo.

5. La normatividad vigente. 


\section{Cuadro 7. Directrices para la remediación de suelos contaminados por hidrocarburos}

Implica ubicar dónde se encuentra la fuente que origina la contaminación; así como conocer la historia de las fugas y derrames accidentales.
Identificación de la fuente de contaminación

Selección de contaminantes y parámetros por considerar

Caracterización del suelo y del subsuelo de los sitios contaminados

Estrategia de muestreo

Definición de niveles de limpieza
Los parámetros que suelen recomendarse como indicadores de la contaminación del suelo por hidrocarburos son los hidrocarburos totales de petróleo (ТPH), los compuestos orgánicos volátiles (Voc), los BTEX (benceno, tolueno, etilbenceno y xilenos) y los hidrocarburos aromáticos policíclicos (РАH).

Este es un paso crucial pues, dependiendo de los resultados, se podrá diseñar el esquema de muestreo de los contaminantes y determinar qué tecnología puede aplicarse. Entre los principales parámetros que deben tenerse en cuenta están: la estratigrafía del sitio, la permeabilidad y porosidad del suelo, la profundidad y el grupo de suelo. Este tipo de datos, junto con los de las propiedades físicas y químicas de los contaminantes (solubilidad en agua, presión de vapor, constante de la ley de Henry y coeficiente de partición octanol/agua), permiten evaluar la toxicidad de exposición de posibles receptores (seres humanos, flora o fauna).

Depende de la relevancia y pertinencia de los datos sobre los niveles de contaminantes y la extensión contaminada por derrames petroleros. En particular, es preciso definir el tipo de muestreo para el suelo en áreas del trópico; primero deben ubicarse los puntos de muestreo y la cantidad de puntos. Igualmente, la metodología de evaluación de riesgos debe centrarse en determinar la exposición del suelo y su posible afectación a los acuíferos y a la liberación de vapores. Bautista-Zúñiga (1999: 99) menciona cinco tipos de muestreo: a juicio, al azar, estratificado, sistemático y compuesto. Con esto se puede elaborar una buena estrategia, relacionando el alcance de los resultados con las áreas contaminadas.

En México, la ausencia de criterios ecológicos y de normatividad en materia de suelos contaminados dificulta la decisión sobre qué tanto debe limpiarse un sitio contaminado. La evaluación de los riesgos asociados a los niveles detectados de contaminantes puede suplir esta deficiencia, al indicar la probabilidad de que ocurran efectos adversos; sin embargo, también en este caso se enfrentan dificultades, pues no se han establecido los criterios de riesgo a seguir en función de los usos presentes y futuros de los suelos restaurados. Asimismo, suele desconocerse el hecho de que la geología de nuestro país no es necesariamente similar a la de los sitios de otros países cuyos niveles de limpieza se toman como referencia.

Tecnologías aplicables a la restauración

En este apartado debe seleccionarse la tecnología más adecuada para el tratamiento del sitio contaminado con hidrocarburos. 


\section{Directrices para la remediación de suelos contaminados}

Las directrices deben permitir la evaluación de riesgos teniendo en cuenta las condiciones locales y el impacto de los suelos contaminados; por consiguiente, su aplicación debe facilitar la comparación internacional de las evaluaciones de los riesgos e impactos ambientales en la contaminación de los suelos (IPIECA, 1991: 15; CAC/GL, 2013: 7). Por ello, poner en práctica estas directrices requiere asistencia profesional y entrenamiento especializado, lo cual facilitará que al usar cualquiera de las biotecnologías seleccionadas estas sean aplicadas con éxito.

La Dirección General de Parques Nacionales y Agencia de Parques de Canadá (2008: 97) asegura que debería tenerse presente el enfoque holístico (la función y la dinámica para la remediación de los suelos contaminados por hidrocarburos), integrando en la toma de decisiones los procesos sociales y culturales. La utilidad de estas directrices se ajusta estrictamente a la medida en que ayuden a recuperar áreas perjudicadas por los derrames de petróleo.

En el cuadro 7 se describe cómo pueden utilizarse las directrices en un contexto de contaminación de suelos por hidrocarburos.

\section{Gestión de suelos contaminados por hidrocarburos}

En la figura 1 se muestra el diagrama general para la gestión de suelos contaminados, considerando criterios específicos de limpieza basados en la evaluación de riesgos.

Bremer-Bremer (1999) señala que más vale prevenir que remediar, pues es mucho más costoso en tiempo, dinero y esfuerzo limpiar el área afectada, que prevenir las afectaciones del entorno natural, al menos a mediano y largo plazo. Los costos de prevención pueden ser presupuestados de las ganancias que los originaron, mientras que los de remediación difícilmente pueden preverse y presupuestarse de esas ganancias, sin tomar en cuenta los efectos negativos en el entorno y en la salud de los afectados.

En los programas de evaluación de los suelos contaminados por hidrocarburos totales del petróleo también hay que considerar tres fases principales, de acuerdo con Motuzova (1994a y 1994b) y Oleschko (1999), estas son: 


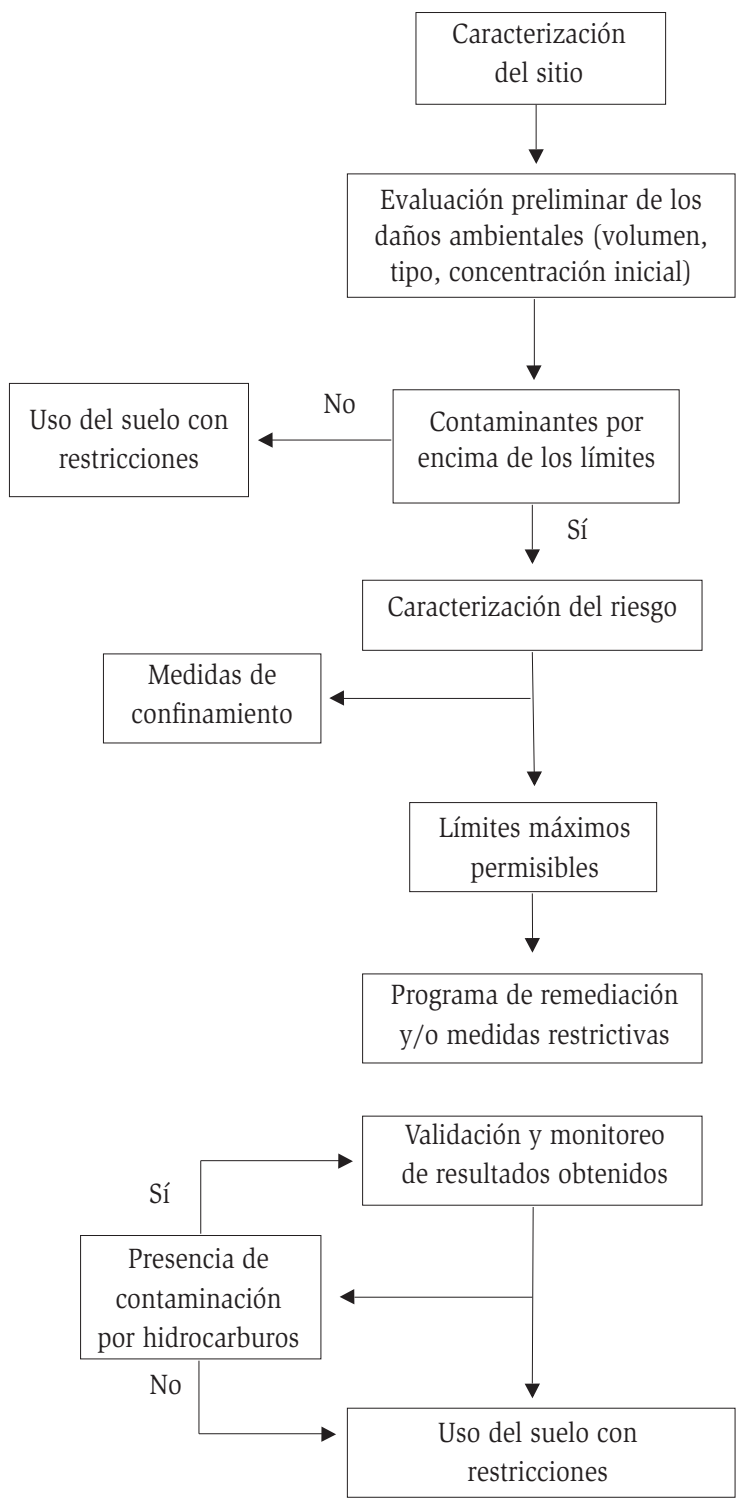

Fuente: Elaboración propia a partir de Saval, 1999 у ссме, 2001: 8. 
- Diagnóstico de la calidad del sistema.

- Pronóstico de su desarrollo en el tiempo y en el espacio.

- Diseño de las prácticas de control y de mejoramiento.

Las dos primeras fases de análisis se complican, por lo que, dada la alta variabilidad en el espacio y a la dinámica en el tiempo en dichos sistemas, Bremer-Bremer (1999) recomienda que se empleen técnicas no destructivas para el análisis de su estructura original.

La lgeEpa (Presidencia de la República, 1996: 27) plantea que una de las principales fuentes de contaminación de los suelos es la disposición inadecuada de los residuos, y establece que restaurar y remediar el área afectada es responsabilidad de quien contamine; por esta razón, es preciso reglamentar y normar las disposiciones de la ley en la materia; de hecho, la Ley Federal de Responsabilidad Ambiental (Presidencia de la República, 2013) avala lo anterior, ya que plasma en su artículo 10 que "toda persona física o moral que con su acción u omisión ocasione directa o indirectamente un daño al ambiente, será responsable y estará obligada a la reparación de los daños, o bien, cuando la reparación no sea posible a la compensación ambiental que proceda, en los términos de la presente ley."

\section{Propuesta coordinada}

Para obtener los mejores resultados en la atención de un área contaminada por hidrocarburos, lo principal es conocer las características del sitio y las del contaminante, esto a través de los muestreos y de los análisis, para posteriormente utilizar la biotecnología de remediación más adecuada.

En este trabajo , en apego a las normas mexicanas e internacionales, se han descrito algunas biotecnologías para conservar y restaurar los suelos contaminados por hidrocarburos del petróleo de manera sustentable. Las biotecnologías existentes para la remediación de suelos afectados por los derrames de petróleo ofrecen varios métodos no destructivos y de bajo costo. La biorremediación y su monitoreo son técnicas distintas.

Se necesitan campañas de difusión y concientización sobre las consecuencias de un suelo contaminado. Para tal fin se requiere reforzar los sistemas de 
educación ambiental sobre los suelos contaminados y así lograr una mayor sensibilización respecto del uso y aprovechamiento del recurso suelo y una mejora en la restauración de las áreas afectadas.

Es necesario tener en claro que debe realizarse la caracterización del sitio y del problema, pues la estrategia de remediación puede ser específica para el grupo del suelo y su tipo la afectación, logrando con ello remediar con una buena efectividad.

\section{Fuentes citadas}

Abraham, E. (2004). "La restauración ambiental: una nueva rama de la economía”. Economía Informa, 328, 89-105.

Adams-Schroeder, R.H. y O. Castillo-Acost (2000). "Fitorremediación y fitomitigación para restauración de instalaciones petroleras”. Kuxulkab'. Revista de Divulgación, 5 (11), 30-34.

Álvarez-Gordillo, G.C. (2011). Educación y gestión del riesgo de desastre: procesos educativos en la Cuenca Alta Grijalva. México: El Colegio de la Frontera Sur/Fondo Regional de Ciencia y Tecnología.

Bautista-Zúniga, F. (1999). Introducción al estudio de la contaminación del suelo por metales pesados. Mérida: Universidad Autónoma de Yucatán.

Bremer-Bremer, M.H. (1999). "Responsabilidad del sector privado en el cuidado y manejo sustentable del recurso suelo", en C. Siebe et al. (eds.). Conservación y restauración de suelos. México: Programa Universitario de Medio Ambiente-Universidad Nacional Autónoma de México (PumAUNAM), 141-160.

CAC/GL. (2013). "Directrices sobre la aplicación de la evaluación de riesgos en los piensos" [en línea]. Principios y Directrices para la Aplicación de la Evaluación de Riesgos Microbiológicos. Unión Europea: Comisión del Codex Alimentarius: Manual de Procedimientos. Disponible en www. codexalimentarius.org/input/download/standards/13322/ CXG 080s.pdf [2014, 23 de agosto].

Carabias-Lillo, J. (1999). "Políticas para evitar el deterioro de los suelos en México”, en C. Siebe et al. (eds.). Conservación y restauración de suelos. México: PUMA-UNAM, 17-25. 
Carmona-Díaz, G. (2012). "Nuevo derrame de petróleo en el manglar del río Coatzacoalcos, Veracruz, México”, en L.E. Amador-del Ángel et al. (eds.). Memorias del Segundo Congreso Mexicano de Ecosistemas de Manglar. Ciudad del Carmen: Universidad Autónoma del Carmen, 3637.

Carpena, R.O. y M.P. Bernal (2007). “Claves de la fitorremediación: fitotecnologías para la recuperación de suelos”. Ecosistemas, 16 (2), 1-3.

cCME (2001). Canada-Wide Standards for Petroleum Hydrocarbons (PHC) in Soil.

Winnipeg: Canadian Council of Ministers of the Environment.

Chan-Quijano, J.G. et al. (2012). "Germinación y sobrevivencia de especies arbóreas que crecen en suelos contaminados por hidrocarburos". Teoría y Praxis 12, 102-119.

Chan-Quijano, J.G. et al. (2013). “Especies vegetales útiles para fitorremediar suelos contaminados con hidrocarburos totales del petróleo: un apoyo para la restauración ecológica”. Boletín Divulgativo de la Red Iberoamericana y del Caribe de Restauración Ecológica, 7 (3), 11-14.

Chiou, C.T., S.E. McGroddy y D.E. Kile (1998). "Partition Characteristics of Polycyclic Aromatic Hydrocarbons on Soils and Sediments”. Environmental Science Technology, 32 (2), 264-269.

Cunningham, S.D., Berti, W.R., Huang, J.W. (1995). "Phytoremediation of contaminated soils and sediments”, en H.D. Skipper y R.F. Turco (eds.). Bioremediation: Science and Applications. Madison: Soil Science Society of America/American Society of Agronomy/Crop Science Society of America, 145-156.

Dirección General de Parques Nacionales y Agencia de Parques de Canadá (2008). Principios y directrices para la restauración ecológica en las áreas protegidas naturales de Canadá. Canadá: El Consejo Canadiense de Parques y Parques Canadá.

D’Luna-Fuentes, A., A. Vargas-Mena y Amezcua y G. Negrete-Fernández (1999). "El ordenamiento territorial y los sistemas de información geográfica como base para el inventario de los recursos naturales”, en C. Siebe et al. (eds.). Conservación y restauración de suelos. México: PUMA-unam, 309-336.

Department of Environment-Government of Nunavut (2009). Environmental Guideline for Contaminated Site Remediation. Nunavut: Environmental 
Protection Division-Department of Environment-Government of Nunavut.

EPA (1997). Rules of Thumb for Superfund Remedy Selection. EPA 540-R-97-013. Washington: United States Environmental Protection Agency.

Etchevers, J.D. (1999). "Indicadores de la calidad del suelo", en C. Siebe et al. (eds.). Conservación y restauración de suelos. México: PUMA-UnAm, 239-261.

Fernández-Linares, L.C. et al. (2006). Manual de técnicas de análisis de suelos aplicadas a la remediación de sitios contaminados. México: Instituto Mexicano del Petróleo/Secretaría de Medio Ambiente y Recursos Natural/Instituto Nacional de Ecología.

Ferrera-Cerrato, R. et al. (2007). "Fitorremediación de un suelo contaminado con combustóleo utilizando Phaseolus coccineus y fertilización orgánica e inorgánica”. Agrociencia, 41 (8), 817-826.

Frausto-Martínez, O. (2008). "Línea de investigación en turismo y desastres naturales: introducción al estudio de las amenazas, riesgos y desastres naturales”, en A. Palafox-Muñoz y O. Frausto-Martínez (coords.). Turismo: desastres naturales, sociedad y medio ambiente. México: Universidad de Quintana Roo/Plaza y Valdés, 15-23.

Frély, R. (2013). Plantas beneficiosas y descontaminantes: para la salud y el medio ambiente. Barcelona: Obelisco.

González-Chávez, M.C.A. (2005). "Recuperación de suelos contaminados con metales pesados utilizando plantas y microorganismos rizosféricos”. Terra Latinoamericana, 23 (1), 29-37.

Hewitt, K. (1997). Geographical Study of Disaster. Nueva York: Allen and Unwin.

Ibáñez-Pérez, R.M. (2012). "Indicadores de sustentabilidad: utilidad y limitaciones”. Teoría y Praxis, 11, 102-126.

IMP (2010). Dirección de seguridad y medio ambiente. México: Instituto Mexicano del Petróleo/Gerencia de Comunicación Social y Relaciones Públicas.

IPIECA (1991). Directrices sobre las consecuencias biológicas de la contaminación por hidrocarburos. Londres: International Petroleum Industry Environmental Conservation Association.

142 Merkl, N., R. Schultze-Kraft y C. Infante (2004). "Phytoremediation of Petroleum Contaminated Soils in the Tropics - Pre-Selection of Plant Species 
from Eastern Venezuela”. Journal of Applied Botany and Food Quality, 78 (3), 185-192.

Moosavi, G.S. y M.J. Seghatoleslami (2013). "Phytoremediation: A Review". Advance in Agriculture and Biology, 1 (1), 5-11.

Motuzova, G.V. (1994a). "Buffering of Soils to External Chemical Inputs”. Eurasian Soil Science, 26 (9), 20-30.

(1994b). "Nature of Soil Buffer Capacity to External Chemical Impacts". Pochvovedenie, 49 (3), 46-52.

Ochoa-Gaona, S. et al. (2011). Estudio prospectivo de especies arbóreas promisorias para la fitorremediación de suelos contaminados por hidrocarburos. Villahermosa: Gobierno del Estado de Tabasco/Secretaría de Recursos Naturales y Protección Ambiental/El Colegio de la Frontera Sur/Petróleos Mexicanos.

Oleschko, K. (1999). "Paradoja tecnológica en la ciencia del suelo nacional: nuevos métodos y técnicas de análisis no destructivos de los sistemas naturales”, en C. Siebe et al. (eds.). Conservación y restauración de suelos. México: PUMA-UnAM, 279-307.

Pérez-Hernández, I.S. et al. (2013). "Tolerance of Four Tropical Ttree Species to Heavy Petroleum Contamination”. Water Air and Soil Pollution, 224 (1637), 1-13.

Plaster, E.J. (2000). La ciencia del suelo y su manejo. Madrid: Paraninfo.

Presidencia de la República (1996). "Ley General del Equilibrio Ecológico y la Protección al Ambiente”. Diario Oficial de la Federación, 13 de diciembre [última reforma DOF 09-01-2015].

Presidencia de la República (2013). "Ley Federal de Responsabilidad Ambiental”. Diario Oficial de la Federación, 7 de junio.

Rivera-Cruz, M.C. (2004). "Biotecnología para descontaminar suelos con hidrocarburos del petróleo crudo”. Tecnociencia Universitaria, 3 (9), 44-55.

Rivera-Cruz, M.C., E. Maldonado-Chávez y A. Trujillo-Narcía (2012). "Effects of Crude Oil on the Growth of Brachiaria mutica and Leucaena leucocephala and on Soil and Plant Macronutrients". Tropical and Subtropical Agroecosystem, 15 (2), 30-39.

Rivera-Cruz, M.C. y A. Trujillo-Narcía (2004). "Estudio de toxicidad vegetal en suelos con petróleos nuevo e intemperizado”. Interciencia, 29 (7), 369-376. 
Saval, S. (1999). "Éxitos y fracasos de la remediación de suelos en sitios contaminados con hidrocarburos”, en C. Siebe et al. (eds.). Conservación y restauración de suelos. México: Puma-unam, 511-526.

Sierra-Villagrana, R. (2006). "Fitorremediación de un suelo contaminado con plomo por actividad industrial”. Tesis de Licenciatura. Coahuila: Universidad Autónoma Agraria Antonio Narro.

Sims, R.C. (1993). "Site Characterization Requirements", en United States Environmental Protection Agency (ed.). Biorremediation of Hazardous Waste Sites: Practical Approaches to Implementation. Washington: United States Environmental Protection Agency, 3-1-3-19.

Velasco Trejo, J.A. y T.L. Volke Sepúlveda (2003). "El composteo: una alternativa tecnológica para la biorremediación de suelos en México”. Gaceta Ecológica, 66, 41-53. 